\title{
SYSTEMS OF EQUATIONS IN THE PREDUAL OF A VON NEUMANN ALGEBRA
}

\author{
MICHAEL MARSALLI
}

(Communicated by Palle E. T. Jorgensen)

\begin{abstract}
A von Neumann algebra $\mathscr{A}$ on a separable, complex Hilbert space $\mathscr{H}$ has property $\mathbf{A}_{n}$ if for every $n \times n$ array $\left\{f_{i, j}\right\}$ of elements in the predual there exists sequences $\left\{x_{i}\right\},\left\{y_{j}\right\}$ in $\mathscr{H}$ such that $f_{i, j}(A)=\left(A x_{i}, y_{j}\right)$ for all $A$ in $\mathscr{A}$ and $0 \leq i, j<n$. We show that the von Neumann algebras with property $\mathbf{A}_{\aleph_{0}}$ are the von Neumann algebras with properly infinite commutant. We describe how these properties are transformed by the tensor product. We characterize the abelian von Neumann algebras with property $\mathbf{A}_{n}$.
\end{abstract}

Let $\mathscr{H}$ be a separable, complex Hilbert space, and let $\mathscr{L}(\mathscr{H})$ be the algebra of bounded linear operators on $\mathscr{H}$. Let $\mathscr{S}$ be an ultraweakly closed subspace of $\mathscr{L}(\mathscr{H})$, and let $\mathscr{S}_{*}$ be the space of ultraweakly continuous linear functionals on $\mathscr{S}$. We call $\mathscr{S}_{*}$ the predual of $\mathscr{S}$. Since the fundamental paper of S. Brown [2], there has been considerable interest in the structure of $\mathscr{S}_{*}$, particularly when $\mathscr{S}$ is a singly generated dual algebra (cf. [1]). But the study of the predual of a von Neumann algebra goes back to Murray and von Neumann in [5]. In this paper we use the ideas in [1] to obtain more precise information about the predual of certain von Neumann algebras, including the type III and abelian ones.

For $x, y$ in $\mathscr{H}, x \otimes y$ will denote the element of $\mathscr{S}_{*}$ defined by $x \otimes y(S)=$ $(S x, y)$ for $S$ in $\mathscr{S}$. The following definition plays a central role.

Definition. Let $\mathscr{S}$ be an ultraweakly closed subspace of $\mathscr{L}(\mathscr{H})$, and let $m$ and $n$ be cardinal numbers with $1 \leq m, n \leq \aleph_{0}$. The space $\mathscr{S}$ has property $\mathbf{A}_{m, n}$ if for every $m \times n$ array $\left\{f_{i, j}\right\}, 0 \leq i<m, 0 \leq j<n$, of elements of $\mathscr{S}_{*}$ there exist sequences $\left\{x_{i}\right\}, 0 \leq i<m$, and $\left\{y_{j}\right\}, 0 \leq j<n$, in $\mathscr{H}$ such that $f_{i, j}=x_{i} \otimes y_{j}$ for $0 \leq i<m, 0 \leq j<n$.

We write $\mathbf{A}_{n}$ for $\mathbf{A}_{n, n}$. Von Neumann algebras with property $\mathbf{A}_{1}$ have been previously characterized, and we reproduce some of these characterizations along with some new ones in Theorem 1. Theorem 4 describes the von Neumann algebras with property $\mathbf{A}_{\aleph_{0}}$. In particular, type III algebras have

Received by the editors December 27, 1989.

1980 Mathematics Subject Classification (1985 Revision). Primary 46L10; Secondary 47D30.

Key words and phrases. Von Neumann algebra, dual algebra, predual. 
property $\mathbf{A}_{\aleph_{0}}$. Theorem 6 shows how these properties are transformed by the tensor product. Finally, Theorem 9 characterizes the abelian von Neumann algebras with property $\mathbf{A}_{n}$. We refer the reader to [3] for the terminology and theory of von Neumann algebras. The following refinement of property $\mathbf{A}_{1}$ will be useful.

Definition. Let $\mathscr{S}$ be an ultraweakly closed subspace of $\mathscr{L}(\mathscr{H})$, and let $r \geq 1$. $\mathscr{S}$ has property $\mathbf{A}_{1}(r)$ if for every $f$ in $\mathscr{S}_{*}$ and for every $s>r$ there exist vectors $x$ and $y$ in $\mathscr{H}$ such that $f=x \otimes y$ and $\|x\|\|y\| \leq s\|f\|$.

Henceforth, $\mathscr{A}$ will denote a von Neumann algebra. If $\mathscr{B}$ is also a von Neumann algebra, we write $\mathscr{A} \cong \mathscr{B}$ to denote that $\mathscr{A}$ is unitarily equivalent to $\mathscr{B}$. It is easy to see that the above properties are preserved by unitary equivalence. Also, the above properties are inherited by ultraweakly closed subspaces of $\mathscr{S}$ by [1, Proposition 2.04]. The following theorem, which is an extension of [4, Theorem 3.5], provides several useful characterizations of von Neumann algebras with property $\mathbf{A}_{1}$. For $x$ in $\mathscr{H},[\mathscr{A} x]$ will denote the norm closure of the linear manifold $\{A x: A$ in $\mathscr{A}\}$. The commutant of $\mathscr{A}$ is denoted by $\mathscr{A}^{\prime}$.

Theorem 1. Let $\mathscr{A}$ be a von Neumann algebra. The following are equivalent.

(a) $\mathscr{A}$ has a separating vector.

(b) $\mathscr{A}^{\prime}$ has a cyclic vector.

(c) For every positive normal form $\phi$ on $\mathscr{A}$, there exists a vector $x$ in $\mathscr{H}$ such that $\phi=x \otimes x$ on $\mathscr{A}$.

(d) $\mathscr{A}$ has property $\mathbf{A}_{1}$.

(e) $\mathscr{A}$ has property $\mathbf{A}_{1}(1)$.

(f) $\mathscr{A}$ has property $\mathbf{A}_{1, \aleph_{0}}$.

(g) For every sequence $\left\{x_{i}\right\}$ in $\mathscr{H}$ with $\sum\left\|x_{i}\right\|^{2}<\infty$, there exists a sequence $\left\{A_{i}\right\}$ in $\mathscr{A}^{\prime}$ and $z$ in $\mathscr{H}$ such that $\left\|A_{i}\right\| \leq 1,\|A z\|^{2}=$ $\sum\left\|A x_{i}\right\|^{2}$ for all $A$ in $\mathscr{A}$, and $A_{i} z=x_{i}$ for $0 \leq i<\infty$.

Proof. The equivalence of (a) and (b) is well known. That (b) implies (c) is [3, Part III, Chapter 1, Theorem 4]. To show (c) implies (d), let $f \in \mathscr{A}_{*}$. By [3, Part I, Chapter 4, Theorem 4] for all $A$ in $\mathscr{A}, f(A)=\phi(U A)$ where $\phi$ is a positive normal form on $\mathscr{A}$ with $\|\phi\|=\|f\|$, and $U$ is a partial isometry in $\mathscr{A}$. So there is a vector $x$ in $\mathscr{H}$ such that $\phi=x \otimes x$ on $\mathscr{A}$. Thus $f(A)=\phi(U A)=(U A x, x)=\left(A x, U^{*} x\right)$ for $A$ in $\mathscr{A}$, and $f=x \otimes\left(U^{*} x\right)$ on $\mathscr{A}$.

We show (d) implies (e). Let $f \in \mathscr{A}_{*}$. Then $f(A)=\phi(U A)$ as above. So $\phi=x \otimes y$ on $\mathscr{A}$ for some $x, y$ in $\mathscr{H}$. Because $\phi$ is a positive form on $\mathscr{A}$, there is a $z$ in $\mathscr{H}$ such that $\phi=z \otimes z$ on $\mathscr{A}$ by [3, Part I, Chapter 4, Lemma 2]. So $f=z \otimes\left(U^{*} z\right)$ on $\mathscr{A}$. Because $\phi$ is positive, $\|\phi\|=\phi(I)$. Thus $\|f\|=\|\phi\|=\phi(I)=z \otimes z(I)=\|z\|^{2} \geq\|z\|\left\|U^{*} z\right\|$, since $U$ is a partial isometry. 
We now show (e) implies (g). If $\left\{x_{i}\right\}$ is a sequence in $\mathscr{H}$ with $\sum\left\|x_{i}\right\|^{2}<\infty$, then the form defined by $f(A)=\sum\left(A x_{i}, x_{i}\right)$ for $A$ in $\mathscr{A}$ is a positive normal form. By hypothesis there exist $x, y$ in $\mathscr{H}$ such that $f=x \otimes y$ on $\mathscr{A}$. As before, the positivity of $f$ implies the existence of a vector $z$ in $\mathscr{H}$ such that $f=z \otimes z$ on $\mathscr{A}$, and $\|f\|=f(I)=z \otimes z(I)=\|z\|^{2}$. Observe that $\sum\left\|A x_{i}\right\|^{2}=\sum\left(A^{*} A x_{i}, x_{i}\right)=f\left(A^{*} A\right)=\left(A^{*} A z, z\right)=\|A z\|^{2}$ for $A$ in $\mathscr{A}$. In particular, $\left\|A x_{i}\right\| \leq\|A z\|$ for each $A$ in $\mathscr{A}$ and $0 \leq i<\infty$. Thus for each $i, 0 \leq i<\infty$, we can define functions $A_{i}$ by $A_{i}(A z)=A x_{i}$. Clearly these functions are bounded linear operators which extend continuously to [ $\mathscr{A} z]$. By defining each $A_{i}$ to be 0 on the orthocomplement of $[\mathscr{A} z]$, we can easily show that $A_{i} \in \mathscr{A}^{\prime} ;\left\|A_{i}\right\| \leq 1$, and $A_{i} z=x_{i}$ for $0 \leq i<\infty$.

In order to show that (e) implies (f), we make use of the fact that (e) implies (g). So let $\left\{f_{i}\right\}$ be a sequence in $\mathscr{A}_{*}$. By hypothesis we can find sequences $\left\{x_{i}\right\},\left\{y_{i}\right\}$ in $\mathscr{H}$ such that $f_{i}=x_{i} \otimes y_{i}$ for $0 \leq i<\aleph_{0}$. Choose a sequence $\left\{r_{i}\right\}$ of positive real numbers such that $\sum r_{i}^{2}\left\|x_{i}\right\|^{2}<\infty$. Since (e) implies (g), we can find a sequence $\left\{A_{i}\right\}$ in $\mathscr{A}^{\prime}$ and a vector $z$ in $\mathscr{H}$ such that $A_{i} z=r_{i} x_{i}$. Thus $f_{i}=\left(r_{i}^{-1} A_{i} z\right) \otimes y_{i}=z \otimes\left(r_{i}^{-1} A_{i}^{*} y_{i}\right)$ for $0 \leq i<\aleph_{0}$.

It is clear that (f) implies (e), and we have (e) implies (g), so (f) implies (g). Finally, we must establish that $(\mathrm{g})$ implies (a). Here we will use the separability of $\mathscr{H}$. Let $\left\{x_{i}\right\}$ be a sequence which is dense in $\mathscr{H}$. Choose a sequence $\left\{r_{i}\right\}$ of positive real numbers such that $\sum r_{i}^{2}\left\|x_{i}\right\|^{2}<\infty$. By hypothesis, we can find a vector $z$ in $\mathscr{H}$ such that $\|A z\|^{2}=\sum r_{i}^{2}\left\|A x_{i}\right\|^{2}$ for all $A$ in $\mathscr{A}$. Now $z$ must be a separating vector, for if $T z=0$ for some $T$ in $\mathscr{A}$, then $\sum r_{i}^{2}\left\|T x_{i}\right\|^{2}=0$. Thus $T x_{i}=0$ for $0 \leq i<\infty$. But $\left\{x_{i}\right\}$ is dense, so $T=0$.

For $1 \leq n \leq \aleph_{0}$, we use $M_{n}(\mathscr{A})$ to denote the algebra of $n \times n$ matrices with entries from $\mathscr{A}$ which are bounded operators on $\mathscr{H}^{(n)}$, the direct sum of $n$ copies of $\mathscr{H}$. The following lemma is a special case of [1, Proposition 2.3]. We state it here for convenience.

Lemma 2. Suppose $\mathscr{A}$ is a von Neumann algebra and $1 \leq n<\aleph_{0}$. Then $\mathscr{A}$ has property $\mathbf{A}_{n}$ if and only if $M_{n}(\mathscr{A})$ has property $\mathbf{A}_{1}$.

We now present a useful necessary condition for von Neumann algebras with property $\mathbf{A}_{n}$ when $n$ is finite.

Lemma 3. Let $\mathscr{A}$ be a von Neumann algebra with property $\mathbf{A}_{n}, 1 \leq n<$ $\aleph_{0}$. For every cyclic projection $E$ in $\mathscr{A}^{\prime}$, there exist $n$ pairwise orthogonal projections in $\mathscr{A}^{\prime}$ each of which is equivalent to $E$.

Proof. Let $E$ be a cyclic projection in $\mathscr{A}^{\prime}$. Then $E$ is the projection onto $[\mathscr{A} e]$ for some $e$ in $\mathscr{H}$. Consider the form $f$ on $M_{n}(\mathscr{A})$ defined by $f\left(\left(A_{i, j}\right)\right)$ $=\sum\left(A_{i, i} e, e\right)$ for $\left(A_{i, j}\right)$ in $M_{n}(\mathscr{A})$. Clearly $f$ is a positive normal form on $M_{n}(\mathscr{A})$. By Lemma $2, M_{n}(\mathscr{A})$ has property $\mathbf{A}_{1}$. By Theorem 1, there exists a vector $\mathbf{x}=\left(x_{1}, \ldots, x_{n}\right)$ in $\mathscr{H}^{(n)}$ such that $f=\mathbf{x} \otimes \mathbf{x}$ on $M_{n}(\mathscr{A})$. It follows 
that $(A e, e)=\left(A x_{i}, x_{i}\right)$ for $A$ in $\mathscr{A}$ and $1 \leq i \leq n$, and $\left(A x_{i}, x_{j}\right)=0$ for $A$ in $\mathscr{A}$ and $i \neq j$. Thus $\|A e\|^{2}=\left(A^{*} A e, e\right)=\left(A^{*} A x_{i}, x_{i}\right)=\left\|A x_{i}\right\|^{2}$ for $A$ in $\mathscr{A}$ and $1 \leq i \leq n$. Define $V_{i}(A e)=A x_{i}$ for $A$ in $\mathscr{A}$ and $1 \leq i \leq n$. Then each $V_{i}$ extends to an isometry from [Ae] onto [Ax $\left.x_{i}\right]$. By defining $V_{i}$ to be 0 on the orthocomplement of $[A e]$, we obtain a partial isometry in $\mathscr{A}^{\prime}$ such that $V_{i}^{*} V_{i}=E$ and $V_{i} V_{i}^{*}=E_{i}$, where $E_{i}$ is the orthogonal projection onto $\left[A x_{i}\right]$. Because $\left(A x_{i}, x_{j}\right)=0$ for all $A$ in $\mathscr{A}$ and $i \neq j$, it follows that $E_{i}$ is orthogonal to $E_{j}$ when $i \neq j$. Thus $E_{1}, \ldots, E_{n}$ are the desired projections.

We are now able to characterize the von Neumann algebras with property $\mathbf{A}_{\aleph_{0}}$. For $A \in \mathscr{A}$ and $1 \leq n \leq \aleph_{0}, A^{(n)}$ will denote the direct sum of $n$ copies of $A$, and $\mathscr{A}^{(n)}$ will denote the set $\left\{A^{(n)}: A \in \mathscr{A}\right\}$. We use $\mathscr{A}^{+}$to denote the set of positive operators in $\mathscr{A}$.

Theorem 4. Let $\mathscr{A}$ be a von Neumann algebra. The following are equivalent.

(a) $\mathscr{A}^{\prime}$ is properly infinite.

(b) $\mathscr{A} \cong \mathscr{A}^{\left(\aleph_{0}\right)}$.

(c) $\mathscr{A}$ has property $\mathbf{A}_{\aleph_{0}}$.

(d) $\mathscr{A}$ has property $\mathbf{A}_{n}$ for $1 \leq n<\aleph_{0}$.

(e) $M_{\aleph_{0}}(\mathscr{A})$ has property $\mathbf{A}_{1}$.

Proof. To show (a) implies (b), assume $\mathscr{A}^{\prime}$ is properly infinite. Then $\mathscr{A}^{\prime} \cong$ $M_{\aleph_{0}}\left(\mathscr{A}^{\prime}\right)$ by [6, Corollary 14]. We obtain (b) by taking the commutant of both sides. That (b) implies (c) is a consequence of [1, Proposition 3.9]. That (c) implies (d) is obvious. We show (d) implies (a). Let $f$ be a nonzero normal trace on $\left(\mathscr{A}^{\prime}\right)^{+}$. Then there is a cyclic projection $E$ such that $f(E)>0$. We show that $f$ is infinite. Fix $n, 1 \leq n<\aleph_{0}$. By Lemma 3, we can find pairwise orthogonal projections $E_{1}, \ldots, E_{n}$ in $\mathscr{A}^{\prime}$ which are each equivalent to $E$. So $\sum E_{i}$ is a projection in $\mathscr{A}^{\prime}$, and there are partial isometries $V_{1}, \ldots, V_{n}$ in $\mathscr{A}^{\prime}$ such that $V_{i}^{*} V_{i}=E$ and $V_{i} V_{i}^{*}=E_{i}$ for $1 \leq i \leq n$. Because $f$ is a trace, $f(E)=f\left(V_{i}^{*} V_{i}\right)=f\left(V_{i} V_{i}^{*}\right)=f\left(E_{i}\right)$ for $1 \leq i \leq n$. Thus $f(I) \geq f\left(\sum E_{i}\right)=$ $n f(E)$. Because $n$ was arbitrary, $f$ cannot be a finite trace. So $\mathscr{A}^{\prime}$ is properly infinite.

We now show that (a) implies (e). Let $\mathscr{M}=M_{\aleph_{0}}(\mathscr{A})$. Then $\mathscr{M}^{\prime} \cong\left(\mathscr{A}^{\prime}\right)^{\left(\aleph_{0}\right)}$ is properly infinite. We already have (a) implies (d), so the result follows.

Finally we establish that (e) implies (d). Since $M_{n}(\mathscr{A})$ is unitarily equivalent to a subalgebra of $M_{\aleph_{0}}(\mathscr{A})$ for each $n, M_{n}(\mathscr{A})$ has property $\mathbf{A}_{1}$ for $1 \leq n<$ $\aleph_{0}$. By Lemma 2, $\mathscr{A}$ has property $\mathbf{A}_{n}$ for $1 \leq n<\aleph_{0}$.

Corollary 5. If $\mathscr{A}$ is a type III von Neumann algebra, then $\mathscr{A}$ has property $\mathbf{A}_{\aleph_{0}}$.

Proof. The commutant of $\mathscr{A}$ must also be type III, and so it is properly infinite. 
We use $\mathscr{A} \otimes \mathscr{B}$ to denote the von Neumann algebra generated by $\{A \otimes B: A$ in $\mathscr{A}, B$ in $\mathscr{B}\}$. The next theorem shows how the property $\mathbf{A}_{n}$ is affected by tensor products.

Theorem 6. Let $\mathscr{A}$ and $\mathscr{B}$ be von Neumann algebras. If $\mathscr{A}$ has property $\mathbf{A}_{m}$ and $\mathscr{B}$ has property $\mathbf{A}_{n}$ for $1 \leq m, n \leq \aleph_{0}$, then $\mathscr{A} \otimes \mathscr{B}$ has property $\mathbf{A}_{m n}$. Proof. By Theorem 4 and Lemma 2, it suffices to show that $M_{m n}(\mathscr{A} \otimes \mathscr{B})$ has property $\mathbf{A}_{1}$. We have $M_{m n}(\mathscr{A} \otimes \mathscr{B}) \cong M_{m}(\mathscr{A}) \otimes M_{n}(\mathscr{B})$. By Lemma 2 or Theorem 4 , both $M_{m}(\mathscr{A})$ and $M_{n}(\mathscr{B})$ have property $\mathbf{A}_{1}$. By Theorem $1,\left(M_{m}(\mathscr{A})\right)^{\prime}$ and $\left(M_{n}(\mathscr{B})\right)^{\prime}$ have cyclic vectors $x$ and $y$, respectively. Since $\left(M_{m}(\mathscr{A})\right)^{\prime} \otimes\left(M_{n}(\mathscr{B})\right)^{\prime} \subset\left(M_{m}(\mathscr{A}) \otimes M_{n}(\mathscr{B})\right)^{\prime}$, it is easy to show that $x \otimes y$ is cyclic for $\left(M_{m}(\mathscr{A}) \otimes M_{n}(\mathscr{B})\right)^{\prime}$. Thus $M_{m n}(\mathscr{A} \otimes \mathscr{B})$ has property $\mathbf{A}_{1}$ by Theorem 1.

For $1 \leq n \leq \aleph_{0}$, we use $I_{n}$ for the algebra of scalars on an $n$-dimensional Hilbert space.

Corollary 7. If $\mathscr{A}$ is a von Neumann algebra with property $\mathbf{A}_{1}$ and $1 \leq n \leq \aleph_{0}$, then $\mathscr{A} \otimes \mathbf{I}_{n}$ has property $\mathbf{A}_{n}$.

Proof. It is easy to see that $\mathbf{I}_{n}$ has property $\mathbf{A}_{n}$ for $1 \leq n \leq \aleph_{0}$.

We now turn our attention to abelian algebras. First we consider algebras of uniform multiplicity. Here the multiplicity completely determines the size of the systems we can solve in the predual.

Theorem 8. Let $\mathscr{A}$ be a maximal abelian von Neumann algebra, and let $1 \leq$ $k \leq \aleph_{0}$. Then $\mathscr{A} \otimes \mathbf{I}_{k}$ has property $\mathbf{A}_{m}$ for $m \leq k$. If $k<\aleph_{0}$ and $\mathscr{A} \otimes \mathbf{I}_{k}$ does not act on the space (0), then $\mathscr{A} \otimes \mathbf{I}_{k}$ does not have property $\mathbf{A}_{m}$ for $m>k$. Proof. Because $\mathscr{A}$ is abelian, it has a separating vector. So by Theorem $1, \mathscr{A}$ has property $\mathbf{A}_{1}$. Thus by Corollary $7, \mathscr{A} \otimes \mathbf{I}_{k}$ has property $\mathbf{A}_{k}$, and the first assertion is now obvious.

To prove the second assertion, assume that $\mathscr{A} \otimes \mathbf{I}_{k}$ has property $\mathbf{A}_{m}$ for some $m>k$. Then $\mathscr{A} \otimes \mathbf{I}_{k}$ has property $\mathbf{A}_{k+1}$. For $1 \leq i \leq k$, let $E_{i}$ be the element of $M_{k}(\mathscr{A}) \cong\left(\mathscr{A} \otimes \mathbf{I}_{k}\right)^{\prime}$ which has $I$ in the $i$ th row, $i$ th column, and 0 elsewhere. It is easy to show that $E_{1}, \ldots, E_{k}$ are pairwise orthogonal equivalent cyclic projections with $\sum E_{i}=I$. By Lemma 3, there exist $k+1$ pairwise orthogonal projections $F_{1}, \ldots, F_{k+1}$ each equivalent to $E_{1}$. Because $M_{k}(\mathscr{A})$ is a finite von Neumann algebra, there is a finite trace $f$ on $M_{k}(\mathscr{A})^{+}$ such that $f\left(E_{1}\right)>0$. Now

$$
k f\left(E_{1}\right)=\sum f\left(E_{i}\right)=f(I) \geq f\left(\sum F_{j}\right)=\sum f\left(F_{j}\right)=(k+1) f\left(E_{1}\right),
$$

which is a contradiction.

We finish with a characterization of the abelian von Neumann algebras with property $\mathbf{A}_{n}$. Here $n$ is determined by the smallest multiplicity that occurs nontrivially in the decomposition of the algebra into pieces of uniform multiplicity. 
Theorem 9. Suppose $\mathscr{A}$ is an abelian von Neumann algebra. Let $\mathscr{A}$ be unitarily equivalent to $\sum \oplus\left(\mathscr{A}_{k} \otimes \mathbf{I}_{k}\right)$, where each $\mathscr{A}_{k}$ is a maximal abelian von Neumann algebra acting on a Hilbert space $\mathscr{H}_{k}$. For $1 \leq n \leq \aleph_{0}$, $\mathscr{A}$ has property $\mathbf{A}_{n}$ if and only if $\mathscr{H}_{k}=(0)$ for all $k<n$.

Proof. Suppose $\mathscr{H}_{k}=(0)$ for $k<n$. Trivially, $\mathscr{A}_{k} \otimes \mathbf{I}_{k}$ has property $\mathbf{A}_{n}$ for $k<n$. If $k \geq n$, then $\mathscr{A}_{k} \otimes \mathbf{I}_{k}$ has property $\mathbf{A}_{n}$ by Theorem 8 . By Lemma 2 or Theorem $4, M_{n}\left(\mathscr{A}_{k} \otimes \mathbf{I}_{k}\right)$ has property $\mathbf{A}_{1}$ for every $k, 1 \leq k \leq$ $\aleph_{0}$. Thus $M_{n}\left(\mathscr{A}_{k} \otimes \mathbf{I}_{k}\right)$ has property $\mathbf{A}_{1}(1)$ for every $k$ by Theorem 1 . Now $M_{n}(\mathscr{A}) \cong \sum \oplus M_{n}\left(\mathscr{A}_{k} \otimes \mathbf{I}_{k}\right)$. Since each summand has property $\mathbf{A}_{1}(1)$, the sum has property $\mathbf{A}_{1}$ by [1, Proposition 2.055]. Thus by Lemma 2 or Theorem 4, $\mathscr{A}$ has property $\mathbf{A}_{n}$.

Now we prove necessity. So assume $\mathscr{A}$ has property $\mathbf{A}_{n}$. Because each $\mathscr{A}_{k} \otimes \mathbf{I}_{k}$ is unitarily equivalent to a subalgebra of $\mathscr{A}$, each $\mathscr{A}_{k} \otimes \mathbf{I}_{k}$ has property $\mathbf{A}_{n}$. If $k<n$, then $k$ is finite and $\mathscr{H}_{k}=(0)$ by Theorem 8 .

\section{REFERENCES}

1. H. Bercovici, C. Foias, and C. Pearcy, Dual algebras with applications to invariant subspaces and dilation theory, CBMS Regional Conf. Ser. in Math., no. 56, Amer. Math. Soc., Providence, RI, 1985.

2. S. Brown, Some invariant subspaces for subnormal operators, Integral Equations Operator Theory 1 (1978), 310-333.

3. J. Dixmier, von Neumann algebras, North-Holland, Amsterdam, 1981.

4. A. Loginov and V. Sulman, Hereditary and intermediate reflexivity of $W^{*}$-algebras, Math. USSR-Izv. 9 (1975), 1189-1201.

5. F. Murray and J. von Neumann, On rings of operators, Ann. of Math. 37 (1936), 116-229.

6. D. Topping, Lectures on von Neumann algebras, Van Nostrand Rheinhold, London, 1971.

Department of Mathematics, Illinois State University, Normal, Illinois 61761 